\title{
A Fabrication Process for Nanopatterns Shrinkage with Variable Sizes for Large Area
}

\author{
Shuhao Si, Lars Dittrich, Martin Hoffmann \\ Micromechanical Systems Group, IMN MacroNano ${ }^{\circledR}$, Technische Universität Ilmenau \\ Germany \\ shuhao.si@tu-ilmenau.de; lars.dittrich@tu-ilmenau.de; martin.hoffmann@tu-ilmenau.de
}

\begin{abstract}
In this paper, a cost efficient process chain is designed to shrink periodic nanopatterns to tunable feature sizes. The feature size of the final patterns can be controlled and adjusted. $100 \mathrm{~mm}$ full wafers featuring square holes with $130 \mathrm{~nm}, 160 \mathrm{~nm}, 190 \mathrm{~nm}$ and $220 \mathrm{~nm}$ feature sizes are fabricated from the original wafer containing circular holes with a diameter of $350 \mathrm{~nm}$. The fabrication chain involves well-known technologies such as etching and soft UV nanoimprint lithography (UV-NIL). Based on a single original master, an intermediate template is fabricated featuring an inversed pyramid pattern using soft UV-NIL and subsequent wet chemical etch. By utilizing the slope of the inversed pyramid structures, the mask on the final substrate can be opened featuring tunable dimensions. Cryogenic etching based on $\mathrm{SF}_{6} / \mathrm{O}_{2}$ chemistry enables the creation of the final shrunk nanopatterns with smooth and vertical profile. The fabrication cycles involve only short imprinting and etch processes coping without costly electron beam writings. Therefore, the original micro- and nanostructure wafer can be shrunk into nanopatterns with tunable feature sizes at a constant pitch in a cost effective manner. The generated periodic nanopatterns can be adopted to the fields of NIL templates, photonic crystals, optics, energy conversion and storage, etc.
\end{abstract}

Keywords: nanofabrication, nanopattern shrinkage, soft UV-NIL, tunable feature size, large area, low cost

\section{Introduction}

Device miniaturization has been in demand based on Moore's Law and the concept of "Beyond Moore's Law" 0-0. The functional structures developed from macro to micro- and nano-range. Periodic nanostructures, such as nano-pillars, cavities, line gratings, rods, and wires, have been great attractions in the field of optics, photonics, MEMS/MOEMS/NEMS, energy, life science, etc. 00. Extended patterning area enables the utilization of such nanostructures from research to industrial applications 0. Electron beam lithography (EBL), laser interference lithography (LIL) are mainly employed to produce new periodic nanopatterns. However, they are limited by the high cost and reasonable throughput when the feature size goes to e.g. sub-500 $\mathrm{nm}$ in large area 0 .

In this paper, the feasibility of fabricating nanopatterns with tunable feature sizes in silicon at wafer-level is presented. A master wafer with periodic nanocavities directly written by EBL is used to necessarily initialize the fabrication process. An intermediate template featuring inversed pyramids is fabricated from the master wafer. Diverse nanopattern replicas with variant feature sizes can be produced utilizing the intermediate template. The fabrication processes cope with a series of soft UV nanoimprint lithography (UV-NIL) and etchings, without requiring electron beam writing. Therefore, it is accomplished at low cost and in a relatively high throughput. The feature size of the new set of nanocavities can be effortlessly tuned during the respective etch process, making it the essential motivation of this work.

The methodology and fabrication process are explained and illustrated in the second section. Corresponding to the processing flow, the fabrication results are shown and discussed in the subsequent sections. In the last part, the potential of this methodology for the fabrication of further types of periodic nanostructures is specified. Additional applications are forecasted as well.

\section{Methodology}

The fabrication process flow to shrink the original master nanopatterns to the aspired patterns with tunable feature sizes is schematically illustrated in Table 1. A master featuring circular cavity patterns is prepared (Step A) by traditional nanostructure patterning technologies such as EBL and IL. Soft polymer-based stamps are replicated from the master (Step 
B) to realize the large area patterning, e. g. $100 \mathrm{~mm}$ wafers by soft UV-NIL. The patterns are transferred from the soft stamp into the resist which is coated onto a $\langle 100\rangle$-orientated silicon substrate. A plasma etch process descums the residual resist and structures the mask on the intermediate template. Thus, the silicon substrate is masked by the identical patterns as the master (Step $C$ ). The intermediate template is chemically etched into inversed pyramids by means of $\mathrm{KOH}$ wet etching $($ Step $D)$. The surface of the inversed pyramid intermediate template is treated with FDTS as ASL for further soft stamp replications (Step $E$ ). The final silicon substrate that comprises a hard mask layer on top is subsequently imprinted by the pyramid featured soft stamp (Step $F$ ).

Table 1: Schematic process flow of the shrinkage of nanopatterns with tunable feature sizes.

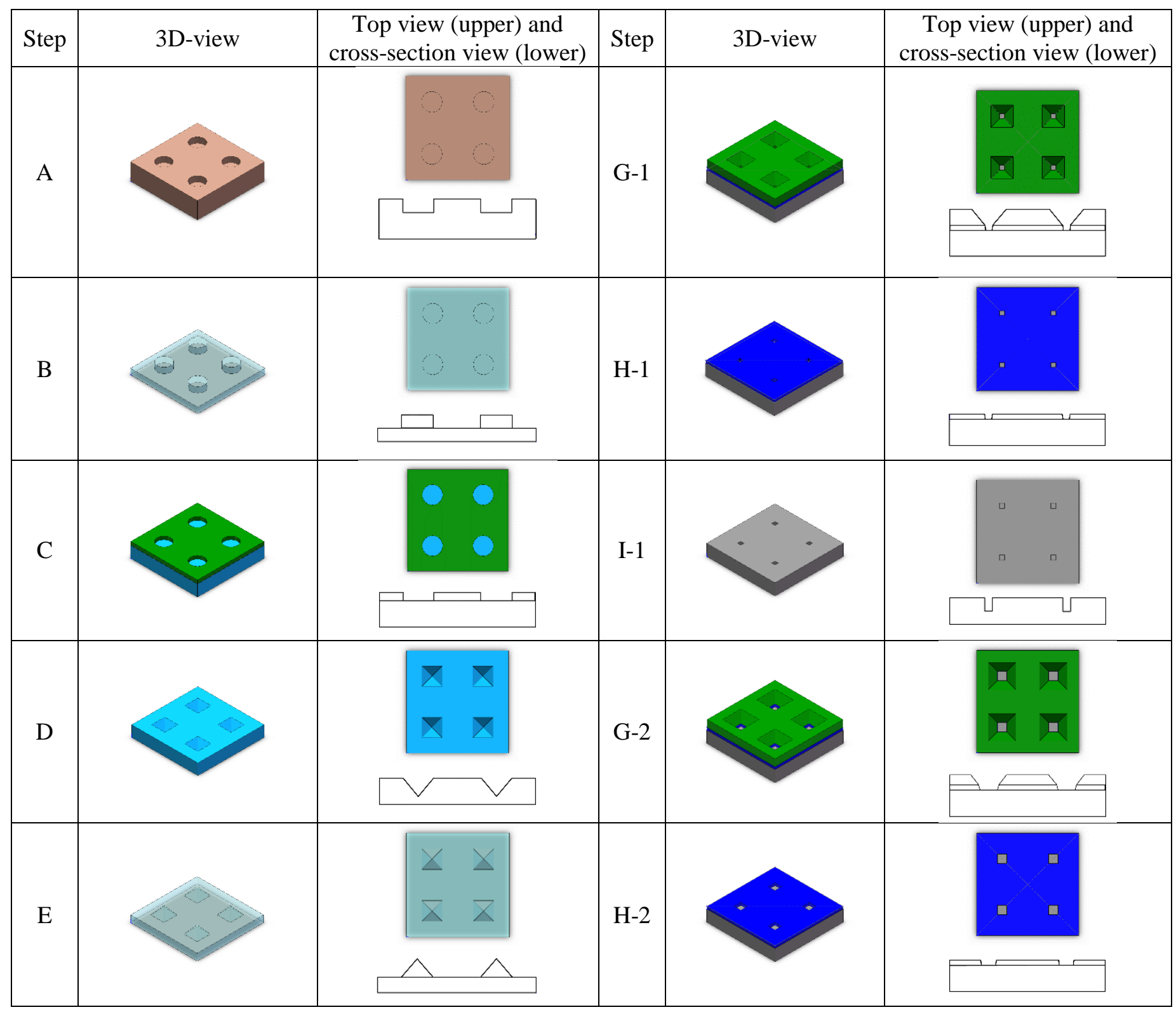






The feature size of the final nanocavities is determined by the dimensions of the etch mask and is defined by the sloped sidewalls of inversed pyramids to obtain the tunable openings. A plasma etch process that is suitable for both the resist and the mask material is chosen to break through the hard mask. By applying different etch durations, the hard mask is opened with tunable sizes (Step $G-1$ and $G-2$ ). As shown in Fig. 1, for instance, the hard mask is opened to the width of $W_{d}$ by applying an etch duration $t$, resulting the height of patterned resist $h_{d}$. By increasing the etch duration, the patterned resist is etched down to the height of $h_{e}$, whereas the width of the opening is correspondingly enlarged to $W_{e}$ due to the sidewall slope. Therefore, openings on the hard mask with various sizes can be controllably achieved.
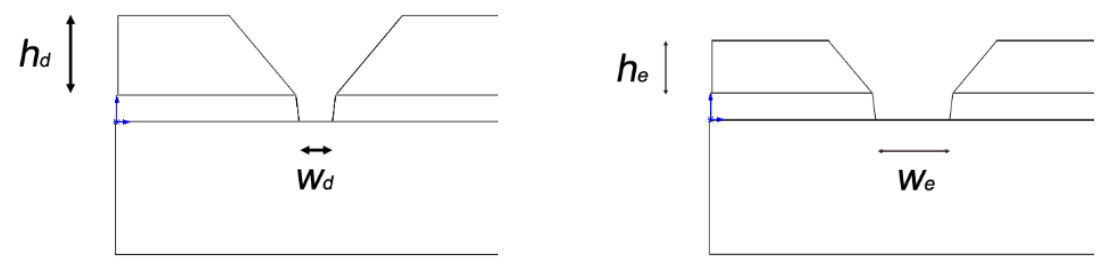

Fig. 1: Schematic diagram of the variant mask openings by different etch durations.

The residual resist can be removed after the masking (Step $H-1$ and $H-2$, respectively). Considering the fact that the pyramid slope in the resist leads to non-vertical sidewalls in the hard mask after plasma etching, the hard mask ought to be preserved as much as possible for silicon substrate etching. The mask should be exposed to the plasma in a short duration to avoid long term chemical reaction and physical bombardment and it is preferably to be protected by extra passivation layers as well. Therefore, the cryogenic silicon etching is employed for the structuring of the final nanocavities. By using the masks patterned in Step $H-1$ and $H-2$, respectively, the circular nanocavities from the original master are shrunk to square nanocavities with tunable feature sizes, as well as smooth and vertical sidewalls (Step $I-I$ and $I-2$, respectively).

\section{Results and Discussion}

A UV-NIL system utilizing soft stamps (GD-N-03, Gdnano Ltd., China) has been employed for imprintings throughout this work. The system takes advantage of the bowing of the soft stamp, thus achieving the conformal contact from the center to the edge of the substrate (center-to-edge scheme) for up to $150 \mathrm{~mm}$ area in ambient atmosphere.

The master used in this work is a $150 \mathrm{~mm}$ silicon wafer patterned by circular nanocavities over the full wafer, featuring a diameter of $350 \mathrm{~nm}$ and a pitch of $500 \mathrm{~nm}$ (cf. Step A). The master is coated with FDTS at $165^{\circ} \mathrm{C}$ in vapor phase for one hour. The FDTS monolayer extensively reduces the surface energy of the silicon in order to enable a gentle demolding of the replicated soft stamp and the master template. Young's equation correlates the surface energy and the contact angle $\theta$ of liquid-gas interface. The contact angle of a water droplet on the FDTS-treated master surface reaches approximately $115^{\circ}$, which corresponds well to the literature data 00.

A hybrid bi-layer soft stamp is designed fitting in the system which is depicted in Fig.2. It consists of a $2 \mathrm{~mm}$ thick PDMS back carrier and a thin feature layer of approx. $150 \mu \mathrm{m}$ toluene-diluted PDMS which carriers the patterns replicated from the master nanocavities (cf. Step $B$ ). It has been reported that lowering the viscosity by toluene improves the wetting behavior and hence increases the patterned height of structures 0 . The fraction of toluene is kept at $20 \mathrm{wt} \%$. To prepare the soft stamp, $20 \mathrm{wt} \%$ toluene is mixed into the PDMS which is prepared at a ratio of 10:1 (base : curing agent) beforehand and degassed thoroughly. The mixture of the toluene-diluted PDMS is spin coated on the surface of the master as feature layer and pre-baked on hotplate at $80{ }^{\circ} \mathrm{C}$ for 30 minutes. When the toluene-diluted PDMS turns to be tacky, the conventional PDMS for the back carrier is cast into a defined replication mold and co-cured with the feature layer at $80{ }^{\circ} \mathrm{C}$ for extra two hours. 
The resist used in the imprinting process is mr-NIL210XP (Micro Resist Technology GmbH, Germany). It is oxygen insensitive and compatible to PDMS-based soft stamps. A $50 \mathrm{~nm} \mathrm{SiO}{ }_{2}$ layer is thermally grown and serves as hard mask on a $100 \mathrm{~mm}<100>$-oriented silicon wafer. After imprinting using the soft stamp, the residual resist is descummed by pure oxygen plasma. The silicon substrate is etched utilizing the patterned $\mathrm{SiO}_{2}$ mask for a wet chemical $\mathrm{KOH}$ etch at a concentration of $40 \%$ and a temperature of $80{ }^{\circ} \mathrm{C}$. The etch rate of silicon in the $\mathrm{KOH}$ solution is approximate $18 \mathrm{~nm} / \mathrm{s}$ whereas the etch rate of $\mathrm{SiO}_{2}$ mask is limited to below $0.2 \mathrm{~nm} / \mathrm{s}$, which results in the selectivity larger than 90:1. The fabricated intermediate template with inversed pyramid patterns after the $\mathrm{SiO}_{2}$ mask removed (cf. Step $D$ ) is shown in Fig. 3.
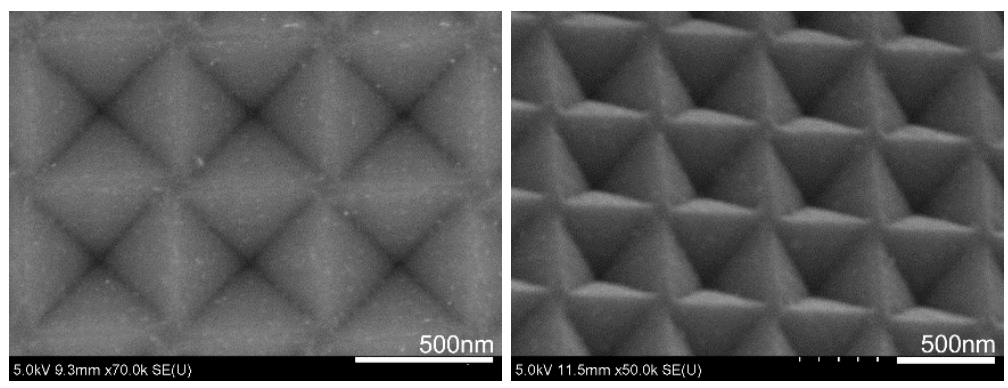

Fig. 2: SEM images of the $100 \mathrm{~mm}$ intermediate template with inversed pyramid patterns: top view (left) and tilted view (right).

Afterwards, the FDTS is deposited on the intermediate inversed pyramid template again to replicate the soft stamps (cf. Step E). $50 \mathrm{~nm} \mathrm{SiO}$ is thermally grown on the silicon substrate as hard mask for the subsequent etching process. Photoresist faces high risks of cracking at cryogenic temperature which is required for the final patterning and the oxygen content in the etching plasma leads to uncontrolled erosion of the mask. Therefore, an intermediate $\mathrm{SiO}_{2}$ hard mask is preferably used to keep the fidelity of patterned surfaces. The wafer is coated with mr-NIL210XP resist for imprinting. The imprinted inversed pyramid structures in resist (cf. Step $F$ ) are shown in Fig. 4.

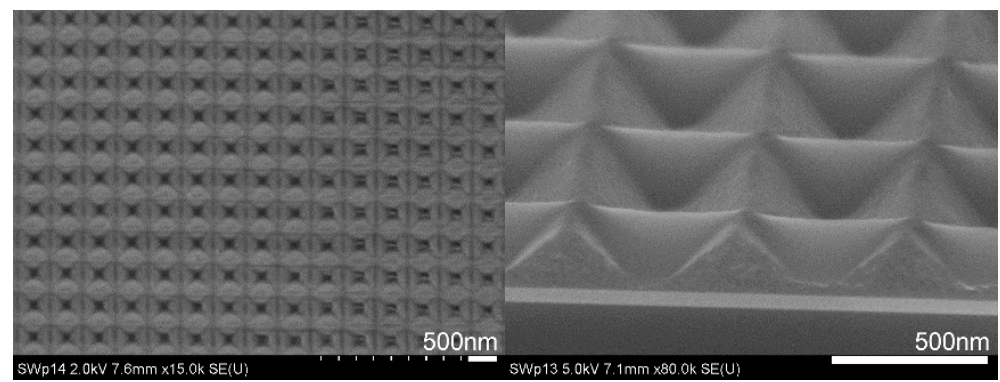

Fig. 3: SEM images of the imprinted inversed pyramid structures: top view (left) and tilted cross-section view (right).

In order to etch down both the patterned resist and open the hard mask, a reactive ion etch (RIE) process utilizing $\mathrm{CHF}_{3}$ and $\mathrm{Ar}$ is used (Plasmalab 100, Oxford Instruments). The etch parameters are compiled in Table 2. As illustrated in Step G-1 and G-2 in Table 1, the lateral dimensions of the hard mask openings can be adjusted by applying different durations of the etch process. Fig. 5a shows that the $\mathrm{SiO}_{2}$ mask is etched to a width of approximate $130 \mathrm{~nm}$ at the bottom for $2 \mathrm{~min}$ and $30 \mathrm{~s}$. The flank angle of the mask, i.e. the angle between the sidewall and the bottom, under such etching conditions reaches nearly $80^{\circ}$. Fig. $5 \mathrm{~b}$ shows that the mask opening is widened to a width of $160 \mathrm{~nm}$ by $2 \mathrm{~min}$ and $50 \mathrm{~s}$ break-through etch. Openings featuring a width of approximate $190 \mathrm{~nm}$ and $220 \mathrm{~nm}$ are obtained by extending the breakthrough etch durations to 3 min $10 \mathrm{~s}$ and $3 \mathrm{~min} 30 \mathrm{~s}$, respectively (Fig. 5c and Fig. 5d). The residual patterned resist is stripped after the break-through etch by applying a microwave oxygen plasma (TePla 200, PVA) at $250 \mathrm{~W}$ for $5 \mathrm{~min}$ (cf. Step $H-1$ and $H-2$ ). The microwave plasma generates a high concentration of active radicals for chemical stripping and low physical bombardment energy such that the $\mathrm{SiO}_{2}$ mask and silicon substrate are not attacked.

Table 2: Process parameters of the break-through etch of the $\mathrm{SiO}_{2}$ mask.

\begin{tabular}{|l|l|l|l|l|} 
Flow rate $(\mathrm{sccm})$ & RF Power $(\mathrm{W})$ & Pressure $(\mathrm{mTorr})$ & Temperature $\left({ }^{\circ} \mathrm{C}\right)$ & Helium Backing \\
\hline
\end{tabular}




\begin{tabular}{|c|c|c|c|c|c|}
\hline CHF3 & $\mathrm{Ar}$ & & & & \\
\hline 12 & 38 & 200 & 50 & 20 & ON \\
\hline
\end{tabular}
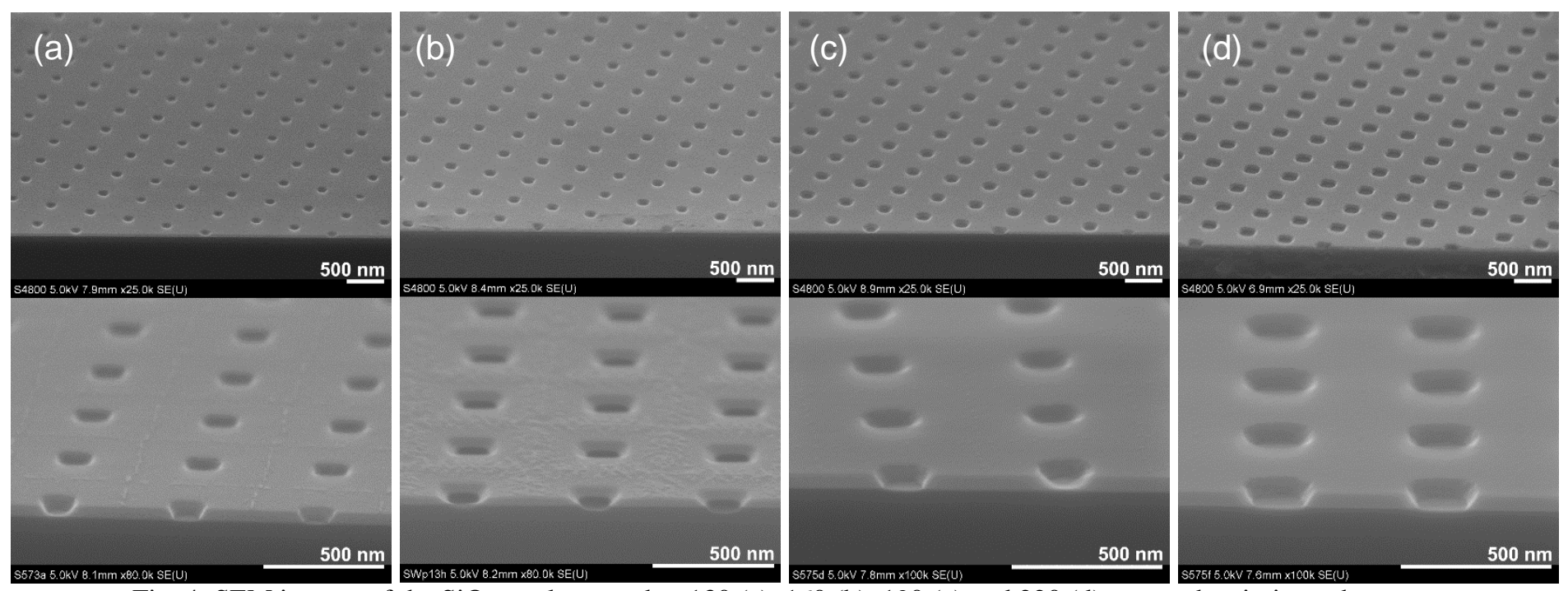

Fig. 4: SEM images of the $\mathrm{SiO}_{2}$ mask opened to 130 (a), 160 (b), 190 (c) and 220 (d) nm on the aiming substrates.

Non-vertical sidewalls are present in the $\mathrm{SiO}_{2}$ mask which is inevitable due to the sloping pyramid ridges in the patterned resist. The etching selectivity of $\mathrm{SiO}_{2}$ to silicon at room temperature does not allow a silicon etching at sufficient aspect ratio without significantly consuming the mask. The consumption of mask leads to reduction of feature sizes for positive structures and expansion of feature sizes for negative structures. In order to enhance the preservation of $\mathrm{SiO}_{2}$ mask, a cryogenic process with liquid nitrogen cooling is employed for silicon etching based on $\mathrm{SF}_{6} / \mathrm{O}_{2}$ chemistry. The cryogenic process makes use of depositing a passivation layer of silicon oxide/fluoride $\left(\mathrm{SiO}_{\mathrm{x}} \mathrm{F}_{\mathrm{y}}\right)$ on the structure sidewalls that prevents them from fluorine radicals attacking. Additionally, the low temperature not only inhibits the radicals physically bombarding the sidewalls but also reduces the chemical consumption of the hard mask. Therefore, the energetic ions mainly penetrate the passivation layer at the bottom and rarely attack the sidewalls. In comparison to the traditional cyclic deep RIE (c-DRIE) process that involves discrete etching and passivation cycles, the cryogenic process promotes the selectivity between the silicon and $\mathrm{SiO}_{2} \mathrm{mask}$ without generating sidewall scallops.

An Inductively Coupled Plasma (ICP) facility (PlasmaPro 100 Cobra, Oxford Instrument) has been used throughout the etching of the final nanocavities. The ICP offers a high density of radicals without generating a large amount of kinetic energy and thus achieves a high etch rate of the silicon substrate. Studies have shown that the temperature and the amount of oxygen will delicately influence the thickness of passivation layer and, therefore, affect the profile of etched structures []. The total flow rate of $\mathrm{SF}_{6}$ and $\mathrm{O}_{2}$ in this case is kept at $50 \mathrm{sccm}$ in which the $\mathrm{O}_{2}$ accounts for $20 \%$. The bottom electrode is powered at an RF frequency of $13.56 \mathrm{MHz}$ for $5 \mathrm{~W}$ and cooled by liquid nitrogen. The electrode temperature is maintained at $-120{ }^{\circ} \mathrm{C}$, and the substrates to be etched are placed in the process chamber for 5 min to level the temperature of substrate to the electrode. The high density plasma is generated independently by the ICP coil at a power of $500 \mathrm{~W}$. The pressure is set to be 6 mTorr for the cryogenic etching. At such low pressure levels, the mean free path length of the ions is larger than the sheath thickness. Therefore, the ions will be hardly deviated from the vertical direction 00. Helium backing is provided for efficient thermal contact. The etch parameters are compiled in Table 3.

Table 3: Process parameters of the cryogenic etching of the shrunken nanopatterns.

\begin{tabular}{|c|c|c|c|c|c|c|}
\hline \multicolumn{2}{|c|}{ Flow rate $(\mathrm{sccm})$} & \multicolumn{2}{|c|}{ Power $(\mathrm{W})$} & \multirow{2}{*}{ Pressure (mTorr) } & \multirow{2}{*}{ Temperature $\left({ }^{\circ} \mathrm{C}\right)$} & \multirow{2}{*}{ Helium backing } \\
\cline { 1 - 4 } $\mathrm{SF}_{6}$ & $\mathrm{O}_{2}$ & $\mathrm{ICP}$ & $\mathrm{RF}$ & 6 & -120 & ON \\
\hline 40 & 10 & 500 & 5 & 6 & \\
\hline
\end{tabular}


The remaining $\mathrm{SiO}_{2}$ mask is completely removed by hydrogen fluoride (HF) wet etch after the cryogenic structuring. The SEM images of the final shrunken nanocavities with feature dimensions of approximate $130 \mathrm{~nm}, 160 \mathrm{~nm}, 190 \mathrm{~nm}$ and $220 \mathrm{~nm}$, respectively, are displayed in Fig. 6a to Fig. 6c. The etch rate according to the conditions in Table 3 reaches approx. $900 \mathrm{~nm} / \mathrm{min}$ and the etch process lasts $15 \mathrm{~s}$. The cryogenic process extensively enlarges the selectivity between the $\mathrm{SiO}_{2}$ mask and the silicon substrate. The $\mathrm{SiO}_{2}$ mask is mostly preserved without obvious consumption and therefore the etched profile does not encounter strong expansions. The gas flow ratio of $\mathrm{SF}_{6} / \mathrm{O}_{2}$ results in a balance of the passivation layer deposition and penetration. Thus, the shrunken square nanocavities possess very smooth and vertical sidewalls, which qualifies them for applications such as NIL templates and photonic crystals.
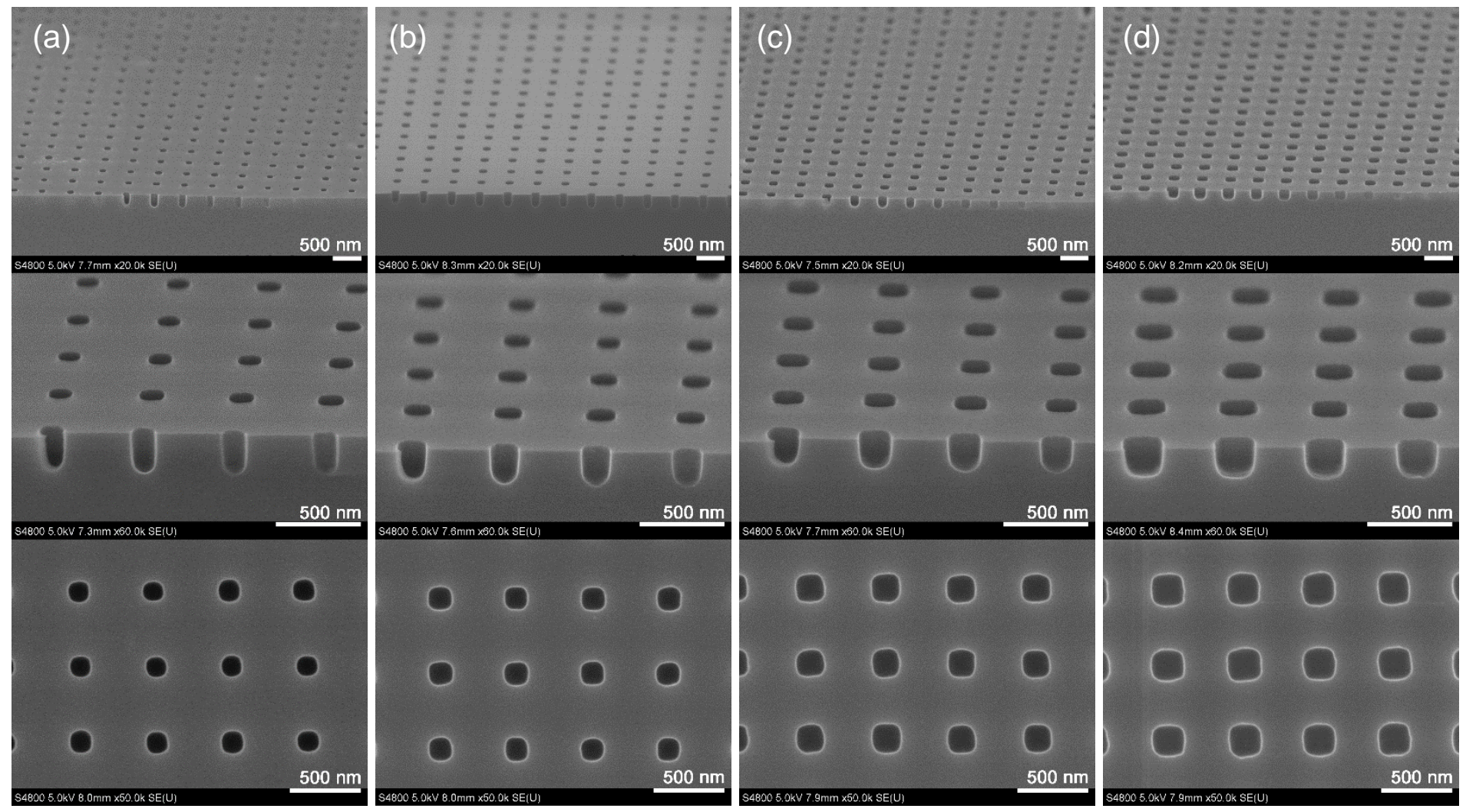

Fig. 5: SEM images of the final nanocavities with the feature size shrunk to approximate 130 (a), 160 (b), 190 (c) and 220 (d) nm.

\section{Conclusion and Outlook}

Based on a single EBL-written master wafer featuring circular nanocavities with a diameter of $350 \mathrm{~nm}$, a shrinkage of nanocavities with feature size down to $130 \mathrm{~nm}, 160 \mathrm{~nm}, 190 \mathrm{~nm}$ and $220 \mathrm{~nm}$ in square layout have been fabricated in this work. The fabrication chain includes a two-stage UV-NIL and etching processes. The production cycle is kept short and simple, and therefore the cost for such fabrication is enormously reduced compared to that written by EBL and such. The introduced method provides a great cost-effective possibility to transfer the nanoscale or microscale patterns into smaller and tunable sizes whereas the pitch of the original patterns is kept constant. By delicately controlling the mask opening step, additional shrinkage of the feature size is acquirable following the same methodology and fabrication processes (Table 4A).

The most essential step in order to shrink the nanopatterns with tunable feature sizes is the fabrication of an intermediate template with sloped sidewalls. A wet chemical KOH etch is employed in the presented case to etch the $\langle 100\rangle$ oriented silicon. Square hole patterns are obtained in the final products following the topology of the pyramids. Alternatively, dry etch approaches can be utilized to fabricate inversed cone profiles instead of $\mathrm{KOH}$-etched inversed pyramids (cf. Step $D$ ). Therefore, following the topology of the cone, the final template can be patterned with circular nanocavities with tunable feature sizes (Table 4B). 
Alternatively, to etch the final template into square hole patterns by means of $\mathrm{SF}_{6} / \mathrm{O}_{2}$ cryogenic process (cf. Step $I-1$ and I-2), the silicon substrate can be etched by $\mathrm{KOH}$ as well to achieve inversed pyramid nanocavities with reduced feature sizes for application-specific purposes (Table 4C).

In case a master template with positive nanostructures such as nanopillars is used, positive intermediate templates can be fabricated featuring either pyramid profile by $\mathrm{KOH}$ etching or cone profile by employing plasma etching. Thus, the final NIL templates can be fabricated into square pillar (Table 4D), pyramid (Table 4E) or circular pillar (Table 4F) patterns with tunable feature sizes.

Table 4: Perspectives of new nanopatterns fabrication using the shrinkage methodology.

\begin{tabular}{|l|l|l|l|l|l|}
\hline & $\begin{array}{c}\text { Master nano- } \\
\text { patterns }\end{array}$ & $\begin{array}{c}\text { intermediate } \\
\text { template }\end{array}$ & shrunken nanopatterns with tunable feature sizes \\
\hline A & & & & & \\
\hline
\end{tabular}

By combining the metal-assisted chemical etching (MaCE) with this methodology, this method provides a great opportunity for broadening the perspectives of nanowires or high aspect ratio nanopillars (Table 4G), with porous, core/shell structures.

\section{Acknowledgements}

The work has been funded within the BMBF-funded project 1D-SENSE (16ES0290). The processing of NIL-related technologies such as soft stamp investigation and plasma etching techniques has been supported by the Institute of Microand Nanotechnologies MacroNano ${ }^{\circledR}$. Special thanks to Dr. Dong Wang, Birgitt Hartmann, Jutta Uziel and Manuela Breiter 
for technological supports. Further appreciations are given to the 5microns GmbH for providing support on the NIL system and process as well as for providing consumables and materials.

\section{References}

[1] G. E. Moore, "Cramming more components onto integrated circuits," Electronics, vol. 38, no. 8, 1965.

[2] D. R. S. Cumming, S. B. Furber, and D. J. Paul, "Beyond Moore's law”, Phil. Trans. R. Soc. A 372, 2013.

[3] The Economist, "After Moore's law," Technology Quarterly, 1203 2016. [Online]. Available: http://www.economist.com/technology-quarterly/2016-03-12/after-moores-law

[4] Q. Zhu, L. Jin, and Y. Fu, "Graded index photonic crystals: A review", Ann. Phys., no. 3-4, pp. 205-218 2015.

[5] S. Murthy, M. Matschuk, Q. Huang, N. K. Mandsberg, N. A. Feidenhans'1, P. Johansen, L. Christensen, H. Pranov, G. Kofod, H. C. Pedersen, O. Hassager, and R. Taboryski, "Fabrication of Nanostructures by Roll-to-Roll Extrusion Coating", Adv. Eng. Mater. vol. 18, no. 4, pp. 484-489, 2015.

[6] H. Hauser, N. Tucher, K. Tokai, P. Schneider, C. Wellens, A. Volk, S. Seitz, J. Benick, S. Barke, F. Dimroth, C. Müller, T. Glinsner, and B. Bläsi, "Development of nanoimprint processes for photovoltaic applications", J. Micro/Nanolith. MEMS MOEMS. vol. 14, no. 3, 031210, 2015.

[7] S. Zhu, H. Li, M. Yang, and S. W. Pang, "High sensitivity plasmonic biosensor based on nanoimprinted quasi 3D nanosquares for cell detection," Nanotechnology, vol. 27, 295101, 2016.

[8] B. Mokaberi, P. Nimmakayala, Z. Ye, P. Schumaker, J. Choi, D. LaBrake, and S. V. Sreenivasan, "Status of jet and flash imprint lithography steppers for sub-25nm semiconductor memory manufacturing", in Proceedings - ASPE 2012 Summer Topical Meeting: Precision Engineering and Mechatronics Supporting the Semiconductor Industry, 2012

[9] H. Schift, "Nanoimprint lithography: An old story in modern times? A review." J. Vac. Sci. Technol. B, vol. 26, no. 2, pp. 458-480, 2008.

[10] M. J. Pellerite, E. J. Wood, and V. W. Jones, "Dynamic contact angle studies of self-assembled thin films from fluorinated alkyltrichlorosilanes," J. Phys. Chem., B, vol. 106, pp. 4746-4754, 2002.

[11] D. Janssen, R. D. Palma, S. Verlaak, P. Heremans, and W. Dehaen, "Static solvent contact angle measurements, surface free energy and wettability determination of various self-assembled monolayers on silicon dioxide," Thin Solid Films, vol. 515, pp. 1433-1438, 2006.

[12] N. Koo, M. Bender, U. Plachetka, A. Fuchs, T. Wahlbrink, J. Bolten, and H. Kurz, "Improved mold fabrication for the definition of high quality nanopatterns by soft UV-nanoimprint lithography using diluted PDMS material," Microelectron. Eng., vol. 84, pp. 904-908, 2007.

[13] AZoNano, "The Cryogenic Process for Etching Micro-Mechanical Systems (MEMS) - Principles, Advances and Applications by Oxford Instruments Plasma Technology," http://www.azonano.com/article.aspx?ArticleID=2739 (accessed 13.03.2016)

[14] M. J. Walker, "Comparison of Bosch and cryogenic processes for patterning high-aspect-ratio features in silicon," in Proc. SPIE 4407, MEMS Design, Fabrication, Characterization, and Packaging, vol. 89, 2001. 